\title{
INVESTIGACIONES
}

\section{Evaluación de la percepción de los niveles de dominio competencial en práctica laboral}

\author{
Evaluation of the perception of competency domain levels in work internship
}

\author{
Harold Tinoco-Giraldo ${ }^{a}$ Eva María Torrecilla Sánchez, \\ Francisco José García Peñalvo ${ }^{a}$ \\ a Universidad de Salamanca, España. \\ htinocogiraldo@usal.es,emt@usal.es, fgarcia@usal.es
}

\begin{abstract}
RESUMEN
Este artículo detalla y analiza las percepciones del nivel de dominio de las competencias profesionales que los estudiantes en práctica laboral del programa de Mercadeo Nacional e Internacional de la Universidad de Manizales (Colombia), desarrollan, fortalecen o registran a partir del reconocimiento de sus tutores de práctica, sus empleadores y ellos mismos. Para efectos de esta investigación se contó con una muestra de estudiantes de sexto semestre en práctica empresarial y se aplicó un cuestionario de evaluación de competencias a partir de los indicadores de dominio. A tal efecto, se parte desde el reconocimiento del dominio competencial a partir del análisis de diferentes prioridades establecidas en dicha práctica. Los resultados ilustran que el nivel de dominio competencial de los estudiantes se encuentra entre el nivel básico y medio, en los cuales empiezan a demostrar las destrezas necesarias para participar de manera efectiva y productiva en sus prácticas laborales.
\end{abstract}

Palabras claves: Competencias profesionales, prácticum, Educación Superior, evaluación competencial.

\begin{abstract}
This article details and analyzes the perceptions of the level of domain of the professional competencies that the students in practicum semester of the National and International Marketing Program of the University of Manizales (Colombia), develop, strengthen or register based on the recognition of their tutors, their employers and themselves. For the purposes of this research, a sample of students enrolled in practice semester was counted and a competency evaluation questionnaire was applied based on the domain indicators. For this purpose, it starts from the recognition of the competence domain from the analysis of different priorities established in the practicum. The results illustrate that the level of proficiency of students is between the basic and middle level, in which they begin to demonstrate the necessary skills to participate effectively and productively in their practicum semesters.
\end{abstract}

Key words: Professional competences, practicum, Higher Education, competence evaluation. 


\section{INTRODUCCIÓN}

La Educación Superior en Colombia está regulada por la normativa Ley 30 de Educación Superior de 1992, en la cual se definen las áreas de conocimiento, los contenidos de los currículos de enseñanza y monitoreo del desarrollo de las habilidades y competencias profesionales en los estudiantes de Educación Superior; elementos que constituyen su objetivo general que es búsqueda de la calidad y mejora del sistema educativo.

Por otra parte, es importante remarcar que dicho objetivo se basa en preparar adecuadamente a los estudiantes para lograr el éxito en sus futuras carreras (Estrada y John, 2012). En este sentido, enfrentar un nivel de rigor académico y adquirir importantes habilidades para la vida pueden considerarse aspectos cruciales de la preparación profesional que reciben los estudiantes mientras están en la Universidad, por esto, cada vez se otorga más importancia al valor del aprendizaje experiencial (Valencia, 2018).

Es aquí, donde las prácticas laborales suponen una oportunidad para desempeñar un papel profesional importante. La importancia de las características de la práctica laboral como el lugar de trabajo, el valor de la tutoría como estrategia de acompañamiento, el valor de la autonomía del practicando al realizar la práctica laboral, la remuneración y tareas significativas en los resultados (Schambach y Dirks, 2002) disertan con la satisfacción profesional de los estudiantes y el dominio de competencias y habilidades (Medina Rivilla y Domínguez Garrido, 2006). Sin embargo, pocos estudios evidencian la relación entre estas características y lo que los empresarios buscan y reciben para sus empresas cuando un estudiante domina una competencia profesional en una situación práctica (Carrera Erazo et al., 2018).

Para reconocerlo sería importante dar respuesta a preguntas como: ¿qué sabemos realmente sobre las prácticas laborales y el impacto en el desarrollo y domino de las competencias profesionales de los estudiantes?, ¿cuáles son esas características más importantes de alcanzar en una práctica laboral para adquirir como resultado positivo el dominio de las competencias necesarias para dicha práctica?

Responder estas preguntas encamina a desarrollar estrategias para la mejora integral de la formación teórico-práctico de estudiantes y disponer de evidencia adicional que indique que las prácticas laborales forman parte de múltiples factores que favorecen la adquisición de competencias profesionales (Álvarez-Santullano y De Prada Creo, 2018). Los procesos de práctica laboral favorecen el desarrollo e incrementan el dominio de competencias y habilidades, lo que puede evidenciarse en condiciones únicas en cada individuo (TinocoGiraldo, 2018) y establecer estándares para la calidad de la práctica laboral.

Es así como este trabajo respalda el impacto que tienen los empleadores y tutores de práctica sobre los resultados de dominio de las competencias profesionales de los estudiantes de práctica laboral en el programa de Mercadeo Nacional e Internacional de la Universidad de Manizales, Colombia. A partir de este estudio, se precisan los comportamientos que los estudiantes de grado de formación superior dominan en situaciones de práctica laboral a partir de un reconocimiento de sus empleadores, tutores asignados en la práctica y de ellos mismos.

También, se realiza una evaluación del grado de dominio por prioridades establecidas en cada competencia y, por último, el estudio del perfil de los atributos necesarios para el desempeño y dominio de dichas competencias. La investigación se estructura de la siguiente manera. En primero, describe la práctica laboral y la formación superior, las competencias en formación y la evaluación de ellas y después, describe el marco metodológico, resultados y conclusiones sobre el estudio. 


\section{LA PRÁCTICA LABORAL Y LA FORMACIÓN SUPERIOR}

Según Zabalza (2016), las prácticas laborales son experiencias de campo que le permiten al estudiante observar y documentar cómo los profesionales que trabajan cumplen con sus responsabilidades laborales. La práctica laboral se concibe como un elemento clave de los currículos universitarios que posibilita el diálogo entre universidad-organización/ institución-estudiante. La función esencial de este elemento formativo radica en formar profesionales capaces de ser transformadores de sus propios procesos, competencias y habilidades profesionales y a su vez dar la oportunidad a los tutores académicos y empleadores de guiar y evaluar su talento.

El propósito de la práctica laboral es facilitar una transición planificada de lo aprendido en aula para aplicarlo al trabajo, y convertirse en la plataforma de conexión entre la universidad y el mundo laboral (Bogdan, 2018). Se busca garantizar el proceso de aprendizaje, teniendo coherencia curricular y apoyo institucional a través de figuras como el tutor de práctica para ayudar al fortalecimiento de competencias y orientación en el campo profesional.

Es importante añadir que la práctica laboral adecúa las tendencias profesionales del estudiante e influye en el logro, desarrollo y buena disposición para ensanchar la brecha entre dominio básico y avanzado de las competencias profesionales de los practicantes (Bermúdez et al., 2011).

La relación entre la conexión de conocimientos técnicos, las habilidades profesionales y la reflexión, les permite a los estudiantes que gradualmente comiencen a desarrollar sabiduría práctica. Sin embargo, para llegar más allá de su nivel personal actual, es necesario ser guiado por alguien que tenga más experiencia. Hay límites en cuanto a la utilidad de la reflexión interna de un estudiante para comprender el razonamiento personal (Navarro Soria et al., 2015). El mentor constituye un modelo para imitar, y la enseñanza se vuelve más como entrenamiento. Por lo tanto, es crucial tener excelentes maestros, tutores y profesionales en la práctica como mentores (Camacho, 2018).

\subsection{LAS COMPETENCIAS EN FORMACIÓN}

La conexión del contenido teórico con la práctica delimita la adquisición de competencias. En este sentido, Charria Ortiz et al. (2011) establecen que desarrollar competencias profesionales va dirigido a la obtención de una capacidad, la que en términos académicos iría encaminada a cumplir una calificación académica. De igual forma, las competencias no solo se desarrollan en el saber educativo; se reflejan también del desempeño práctico del individuo.

En las prácticas laborales del programa de Mercadeo Nacional e Internacional, las competencias van relacionadas con el dominio de procesos productivos en las empresas. En este contexto, el acercamiento a la realidad propia del estudiante mediante la práctica es directamente influenciado por el dominio de sus competencias profesionales y, además, vela por la reconceptualización de su propia formación académica (Martínez Carrillo y Peña Pinzón, 2013).

Este estudio tiene como referencia a Arias (2015) y su agrupación de competencias a partir de los resultados del Proyecto Tuning (2006), se han seleccionado las siguientes competencias como fundamentales en la práctica empresarial: 
1) Gestión de proyectos, se relaciona con las metodologías para organizar un proyecto.

2) Resolución de problemas, implica la identificación y análisis de situaciones problemáticas y su disposición para el encuentro de soluciones efectivas.

3) Pensar de forma crítica, lógica y creativa, permite, el reconocimiento de verdades con demostración lógicas y reales, dividir complejidades en simplicidades y encontrar soluciones a los retos.

4) Trabajo autónomo, capacidad que un estudiante de práctica tiene para autogestionarse, planificar, organizar y priorizar su propio trabajo.

5) Aprender a aprender, reconoce el compromiso que los estudiantes de práctica poseen con el desarrollo de su propio conocimiento y sustitución del mismo, a partir de sus aprendizajes, creencias y experiencias.

6) Comunicar ideas de forma efectiva y el uso de otro idioma, capacidad del estudiante para asociar el logro de objetivos y metas mediante la comunicación verbal y no verbal, tecnológica, sensorial y demás, familiarizándose con los diferentes niveles y características de una situación.

7) Trabajo en equipo, indica la aceptación y respeto por parte de los estudiantes de normas, que facilitan interacción dentro de un determinado grupo en su práctica empresarial.

Para dimensionar el nivel de dominio de estas competencias, se optó por conceptualizarlas desde prioridades específicas para su cumplimiento a partir de ascendencia básica, media y avanzada (Dreyfus y Dreyfus, 2005) (Tabla 1.). El criterio de dominio describe de forma detallada las tareas que deben realizarse y los estándares de calidad esperados en el proceso de práctica, y se integra a los criterios de desempeño, con los comportamientos habituales, características y habilidades de los estudiantes en la potencialización de sus competencias profesionales (Ceja Oseguera et al., 2018).

Tabla 1. Dominio de competencias

\begin{tabular}{|l|l|}
\hline Dominio de competencia & \multicolumn{1}{c|}{ Conceptualización } \\
\hline Básico & $\begin{array}{l}\text { El titular de la competencia puede interpretar y evaluar el conocimiento. } \\
\text { Puede tanto comunicarlo como presentar argumentos coherentes. }\end{array}$ \\
\hline Medio & $\begin{array}{l}\text { El titular de la competencia tiene una comprensión y un análisis críticos } \\
\text { del conocimiento. Puede aplicar el conocimiento. }\end{array}$ \\
\hline Avanzado & $\begin{array}{l}\text { El titular de la competencia es capaz de autogestionarse, con una } \\
\text { comprensión crítica y sistemática del conocimiento. Puede emitir } \\
\text { juicios y proponer soluciones. }\end{array}$ \\
\hline
\end{tabular}

Fuente: Elaboración propia a partir de Dreyfus y Dreyfus (2005). 


\subsection{LA EVALUACIÓN DE COMPETENCIAS PARA LA MEJORA EMPRESARIAL}

Un papel importante de la práctica es proporcionar una base sólida para el desarrollo de una carrera profesional, de ahí, que el factor con el mayor impacto para crear una visión profesional es la evaluación del dominio de las competencias profesionales (RomeroMartín et al., 2017). Lo que experimentan los estudiantes en su práctica crea su visión de la profesión. Por lo tanto, es esencial que a los estudiantes se les ofrezcan prácticas de calidad. Para aprender de la experiencia de campo es necesario volver a mirarla, reconstruirla y evaluarla a través de la interacción entre el individuo, sus alcances profesionales y su aporte a la mejora organizacional.

Para conseguir estas capacidades es necesario adquirir, desarrollar, dominar y expresar conocimientos en el saber, el hacer y el saber hacer (Saldarriaga Ríos et al., 2016). Por otro lado, el reconocimiento de competencias profesionales permite la realización de la práctica profesional acorde a las exigencias actuales de los empleadores (Aznar-Díaz et al., 2018).

Las competencias se manifiestan a través comportamientos relacionados con el desempeño individual, y, a su vez, construyen los requerimientos deseados en un contexto práctico determinado (Chávez et al., 2016). De esta manera, la evidencia muestra que cuando el dominio de competencias profesionales es el resultado de una postura positiva, personal y profesional, las posibilidades de éxito en el desempeño de la práctica laboral son más elevadas (Montoya, 2009; Lentzen, 2016).

Siendo así necesario la puesta en acción de procesos evaluativos donde los empresarios, entes académicos y los mismos estudiantes valoren responsablemente los niveles de dominio de las competencias profesionales en su entorno práctico y así, conjuguen los resultados con las determinaciones personales de los estudiantes, los resultados del análisis realizado y la valoración de desempeño hacia sus conocimientos, habilidades y posibilidades (Tejada Fernández, 2005; Marín et al., 2012). Un sistema de evaluación de las competencias permite determinar si el estudiante domina realmente cuáles son sus tareas y funciones, cómo las desempeña, cuánto conoce de ellas, qué recursos utiliza para poner en práctica lo que sabe y cuál es el aporte al desempeño de las metas de la práctica laboral.

De igual forma la evaluación de competencias facilita un diagnóstico de las fortalezas de los participantes para ser consolidadas y de las debilidades para ser convertidas en oportunidades de mejora e implementar estrategias y acciones encaminadas a cerrar las brechas identificadas y optimizar el desempeño del estudiante. (Véliz Martínez, Jorna Calixto y Berra Socarrás, 2016). La utilización de dichas estrategias promoverá, desarrollará y proporcionará la realización de aprendizajes que superen las metas ya logradas y formen las competencias profesionales que se requieren en la práctica laboral.

\section{MÉTODO}

\subsection{PROPÓSITO DEL ESTUDIO}

En este trabajo se presentan los resultados desde el reconocimiento y oportunidades de interacción del nivel de dominio de las competencias profesionales en práctica laboral de los estudiantes del programa de Mercadeo Nacional e Internacional de la Universidad de Manizales. 
El propósito de esta investigación es reconocer desde los 3 agentes de participación de prácticas laborales (estudiantes de práctica, empleadores y tutores académicos) el dominio que poseen los estudiantes de práctica de sus competencias profesionales, para así, brindar pautas y crear estrategias que aborden de manera integral la verificación dicho nivel de dominio. En definitiva, que exista un reconocimiento del nivel competencial que ha adquirido el estudiante para su beneficio en el sentido formativo. Para tal propósito se ha utilizado un formulario que busca a través de un enfoque científico interdisciplinario, desarrollar una guía para la identificación del dominio de las competencias necesarias en el área de mercadeo. Además, a través de indicadores relevantes de práctica laboral, puede establecer una base sólida y confiable par la obtención de la información (Cano García, 2008).

\subsection{PARTICIPANTES}

La población invitada a participar en el estudio corresponde al número de estudiantes matriculados en sexto semestre del programa de Mercadeo Nacional e Internacional de la Facultad de Ciencias Contables, Económicas y Administrativas de la Universidad de Manizales (2018-II) y que están en práctica laboral I y que coinciden con la muestra a utilizar en el proyecto $\left(\mathrm{N}_{1}=36\right)$, de igual forma se invitaron a participar los Empleadores que sirvieron de vínculo administrativo y direccional con estos estudiantes en su práctica empresarial $\left(\mathrm{N}_{2}=36\right)$ y los Tutores de prácticas $\left(\mathrm{N}_{3}=9\right)$ miembros de la Facultad de Ciencias Contables, Económicas y Administrativas de la Universidad de Manizales, que sirvieron como asesores de prácticas. El número de tutores es menor ya que cada tutor atiende y asesora entre 5 y 7 estudiantes de práctica.

El muestreo seleccionado es no probabilístico, directamente es intencional o de conveniencia (Casal y Mateu, 2003). Para el marco de muestreo se acudieron a las bases de datos de los estudiantes y empresarios administradas por la coordinación de prácticas de la universidad.

\subsection{INSTRUMENTO}

La recogida de información del nivel competencial de los estudiantes se ha llevado a cabo a partir del instrumento Cuestionario de correspondencia de características de competencias, elaborado por Arias (2015), la Figura 1., muestra un ejemplo del formulario en una competencia.

La selección de este instrumento se debe a que no se han encontrado instrumentos que hayan sido diseñados expresamente para obtener los datos requeridos sobre las competencias profesionales específicas en prácticas empresariales y orientados hacia el área de mercadeo.

El cuestionario contiene 8 secciones con un total de 140 ítems. La primera parte incluye las rúbricas, explicaciones del uso del formulario y aspectos sociodemográficos (género, edad, actividad social de la empresa de práctica, entre otros.). A partir del segundo bloque hasta el octavo se centra en cada competencia y los indicadores relacionados por cada una de ellas.

La Figura 2. muestra cómo se adaptaron a partir de Alcaraz Espín et al. (2013) y se relacionaron cada una de las competencias evaluadas, con prioridades relacionadas y específicas para la determinación de los niveles de dominio. 
Figura 1. Ejemplo del cuestionario en la competencia gestión de proyectos

\title{
Cuestionarios de la evaluación de competencias a partir de los indicadores de dominio
}

\begin{abstract}
Dentro de la evaluación para determinar los temas necesarios para el proceso de e-mentoring. Este cuestionario va dirigido a los empresarios y tutores de práctica empresarial. Para determinar los niveles de desarrollo, conocimiento, experiencia y expectativas académico-práctica en cada competencia. La evaluación será medida en la escala:
\end{abstract}

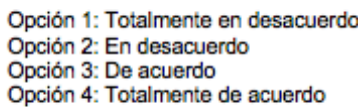

\begin{tabular}{|c|c|c|c|c|c|}
\hline \multicolumn{6}{|c|}{  } \\
\hline \multirow{2}{*}{ Básico } & Planificación básica con dificultades para estableces prioridades. & 1 & 2 & 3 & 4 \\
\hline & Los plazos para las tareas son poco realistas. & 1 & 2 & 3 & 4 \\
\hline Medio & Establece plazos de acuerdo con las prioridades y la carga de trabajo. & 1 & 2 & 3 & 4 \\
\hline \multirow{2}{*}{ Avanzado } & Es capaz de identificar problemas imprevistos y reasignar tiempos a las tareas. & 1 & 2 & 3 & 4 \\
\hline & Establece plazos factibles. & 1 & 2 & 3 & 4 \\
\hline Indicador & \multicolumn{5}{|l|}{ Trabajar de forma sistemática y ordenada. } \\
\hline \multirow{2}{*}{ Básico } & Incumple los plazos asignados a las subtareas/entregables. & 1 & 2 & 3 & 4 \\
\hline & Los resultados generados en el plazo establecido son incompletos. & 1 & 2 & 3 & 4 \\
\hline \multirow{2}{*}{ Medio } & Entrega parte de los resultados en el orden prefijado. & 1 & 2 & 3 & 4 \\
\hline & Los resultados generados en el plazo establecido son completos y de calidad. & 1 & 2 & 3 & 4 \\
\hline \multirow[b]{2}{*}{ Avanzado } & Entrega los resultados completos y en el orden prefijado. & 1 & 2 & 3 & 4 \\
\hline & $\begin{array}{l}\text { Justifica desviaciones del orden o secuencia apropiada planteando alternativas } \\
\text { justificadas. }\end{array}$ & 1 & 2 & 3 & 4 \\
\hline Indicador & \multicolumn{5}{|l|}{ Capacidad para buscar información. } \\
\hline Básico & $\begin{array}{l}\text { Sólo es capaz de realizar búsquedas básicas y no tiene conciencia del valor de la } \\
\text { información obtenida ni de la fuente. }\end{array}$ & 1 & 2 & 3 & 4 \\
\hline Medio & Es capaz de realizar búsquedas de cierta complejidad y encontrar información relevante. & 1 & 2 & 3 & 4 \\
\hline Avanzado & $\begin{array}{l}\text { Es capaz de realizar búsquedas avanzadas en múltiples fuentes, con plena conciencia } \\
\text { del valor de la información obtenida y de la fuente. }\end{array}$ & 1 & 2 & 3 & 4 \\
\hline Indicador & \multicolumn{5}{|l|}{ Capacidad para aplicar y utilizar la información. } \\
\hline Básico & $\begin{array}{l}\text { Presenta información que no se adecúa a los requisitos o se limita a efectuar una mera } \\
\text { descripción de resultados. }\end{array}$ & 1 & 2 & 3 & 4 \\
\hline Medio & $\begin{array}{l}\text { La información encontrada se adecúa a los requisitos, pero no es capaz de aplicarla } \\
\text { eficazmente en los problemas o tareas resolver. }\end{array}$ & 1 & 2 & 3 & 4 \\
\hline Avanzado & $\begin{array}{l}\text { La información encontrada se adecúa a los requisitos, es capaz de aplicarla eficazmente } \\
\text { en los problemas o tareas resolver y ofrece los fundamentos teóricos la sustentan. }\end{array}$ & 1 & 2 & 3 & 4 \\
\hline
\end{tabular}

Fuente propia. Elaborado a partir de Arias (2015).

Los grados de dominio del desempeño de cada estudiante por competencia se atribuyen para reconocer en qué medida el estudiante es competente en el desarrollo práctico de una competencia determinada. Así, el dominio de dichas competencias se exterioriza a través de las labores que cada estudiante de práctica realiza en una situación y proceso determinados (desempeño de la competencia) (Fernández \& Bueno, 2015). De esta forma se determina el nivel puntual de alcance de la competencia en la que se encuentra el estudiante. El formulario está diseñado en escala Likert (Bozal, 2006), con un formato en el cual las respuestas son puntuadas en un rango de 4 opciones de valores referidas a nivel de acuerdo ${ }^{1}$. 
Figura 2. Resumen de las competencias prioridades

\begin{tabular}{|c|c|}
\hline COMPETENCIAS & PRIORIDADES \\
\hline $\begin{array}{l}\text { Gestión de } \\
\text { proyectos }\end{array}$ & $\begin{array}{ll} & \text { Establecer prioridades de objetivos y tareas } \\
\text { Trabajar de forma sistemática y ordenada } \\
\text { - } & \text { Capacidad para buscar información } \\
\end{array}$ \\
\hline $\begin{array}{c}\text { Resolución de } \\
\text { problemas }\end{array}$ & $\begin{array}{ll} & \text { Identificación del problema y análisis de sus causas } \\
\text { : } & \text { Aplicación de un procedimiento efectivo para resolver el problema } \\
\text { - } & \text { Identificación de conflictos y análisis del problema } \\
\text { Toma decisiones y plan de acción }\end{array}$ \\
\hline $\begin{array}{l}\text { Pensar en forma } \\
\text { critica, lógica y } \\
\text { creativa }\end{array}$ & $\begin{array}{l}\text { Establece objetivos concretos para la situación que se le plantea; identifica y valora la } \\
\text { información necesaria para alcanzar esos objetivos } \\
\text { Procesa adecuadamente la información y elabora un plan coherente para resolver la } \\
\text { situación } \\
\text { - Capacidad de generar soluciones originales } \\
\text { - Capacidad para expresar, transmitir y poner en práctica ideas originales }\end{array}$ \\
\hline $\begin{array}{c}\text { Trabajo } \\
\text { autónomo }\end{array}$ & $\begin{array}{ll}\text { Emprender proyectos ambiciosos que implican decisiones } \\
\text { - } & \text { Orientación hacia la innovación y la investigación tecnológica } \\
\text { - } & \text { Capacidad para realizar una buena gestión económica, social y ambiental }\end{array}$ \\
\hline $\begin{array}{l}\text { Aprender a } \\
\text { aprender }\end{array}$ & $\begin{array}{l}\text { - Identifica los elementos más significativos, los agrupa en categorias afines y establece } \\
\text { relaciones de dependencia } \\
\text { Expresa las conclusiones y presenta la información utilizando la técnica más adecuada } \\
\text { Integra conocimientos de diferentes asignaturas para la comprensión del problema o } \\
\text { sistema de estudio } \\
\text { Sintetiza los fundamentos cientifico-tecnológicos del problema y compara las diferentes } \\
\text { soluciones posibles en función de la dinámica o posible solución del problema }\end{array}$ \\
\hline $\begin{array}{l}\text { Comunicar ideas } \\
\text { de forma efectiva } \\
\text { y otro idioma }\end{array}$ & $\begin{array}{l}\text { Expresa sus ideas de forma estructurada y clara } \\
\text { llustra sus ideas integrando ejemplos, analogias, metáforas y otros recursos } \\
\text { adecuadamente. Se ayuda de algún medio de apoyo para sus presentaciones } \\
\text { Responde a las preguntas que se le formulan para defender sus ideas. Manejando con } \\
\text { eficacia la comunicación verbal y no verbal } \\
\text { Fomenta la participación de sus oyentes y pregunta de forma constructiva para conseguir } \\
\text { diálogo y asi mejorar la comunicación de sus ideas }\end{array}$ \\
\hline Trabajo en grupo & $\begin{array}{ll}\text { - } & \text { Capacidad de liderar } \\
\text { Aporte personal y disposición para el trabajo } & \text { Implicación e integración en el equipo }\end{array}$ \\
\hline
\end{tabular}

Fuente. Elaboración propia a partir de Alcaraz Espín et al. (2013).

\subsection{ANÁLISIS DE LOS DATOS}

Con el fin de dar respuesta al objetivo de estudio, se lleva a cabo un análisis descriptivo mediante el porcentaje de acuerdo, la desviación típica y la media en cada una de las dimensiones de las competencias que componen el cuestionario. El análisis se ha apoyado en el programa estadístico SPSS Statistics versión 25 que ha facilitado el análisis de datos.

\section{RESULTADOS}

Los resultados de este estudio se ordenan de la siguiente manera, primero el nivel de percepción competencial de acuerdo con los empresarios, segundo el nivel de percepción competencial de acuerdo con los tutores académicos, tercero el nivel de autopercepción competencial de acuerdo con los estudiantes y, por último, una comparación del nivel de relación de competencias por todos los agentes de participación. 


\subsection{NIVEL DE PERCEPCIÓN COMPETENCIAL DE ACUERDO CON LOS EMPRESARIOS}

Las competencias requeridas por los empresarios que solicitan practicantes a la Universidad de Manizales en el programa de Mercadeo Nacional e Internacional tienen una relación directa con el logro de resultados de calidad y competitividad organizacional que los empresarios buscan con las prácticas. Los empresarios naturalmente buscan en los practicantes una serie de atributos que se ajusten con los requerimientos de la empresa. Y aunque cada empresario determina las características que busca en un practicante, existen características que se repiten, en los resultados encontrados. La información de la Tabla 2., refleja que un $86 \%$ de los empresarios consideran que el nivel de dominio es bajo de la competencia gestión de proyectos y más del $60 \%$ manifestó que las prioridades que los estudiantes deben desempeñar para dominar dicha competencia, se ve reflejada de manera básica con dificultades para estableces dichas prioridades. También revela que en la competencia resolución de problemas, los indicadores identificación del problema, análisis de sus causas, y la aplicación de un procedimiento efectivo para resolver el problema es muy limitado, vinculándo su dominio con un nivel bajo que oscila alrededor de un $77 \%$. Por otro lado, los empresarios perciben en los practicandos una capacidad media de identificación de información necesaria y utilizan criterios capaces de identificar toda la información para valorarla de forma crítica, lógica y creativa. Es destacable que entre el $72 \%$ y $88 \%$ consideran que los estudiantes poseen un nivel medio para generar soluciones originales y están medianamente capacitados para comunicar y transmitir de forma efectiva sus ideas creativas. En cuanto a la competencia trabajo autónomo, aún queda mucho por dominar, pues la percepción de un $6 \%$ de empleadores reflexionan acerca de la carencia de iniciativa propia para poner en marcha proyectos y la puesta en capacidad de gestión para la búsqueda de alternativas introduce alternativas a los métodos tradicionales utilizados. Es importante enfatizar que sólo un $87 \%$ contemplan que los estudiantes tienen un acercamiento mínimo propio al hacia su responsabilidad autónoma de trabajo y a la creación elementos estratégicos en su práctica laboral. Un 94\% de empresarios destacan un dominio medio de la competencia aprender a aprender, y consideran que sería escencial incrementar porcesos hacia la elaboración modelos globales que integren conocimientos de diferentes asignaturas para la comprensión del problema o sistemas de estudio. Es importante guiar los esfuerzos a la mejora de la competencia en la comunicación de ideas efectivas y acertar en la utilización verbal y escrita constate de otro idioma. Un 86\% de los empresarios, consideran que aún falta bastante por lograr para enfatizar la utilización de los medios de apoyo claves en otro idioma. En trabajo en equipo, se demostró una disposión media por mejorar e implicarse e integrase en el equipo de trabajo, de un $61 \%$ a un $83 \%$ de empleadores están satisfechos con esta competencia.

\subsection{NIVEL DE PERCEPCIÓN COMPETENCIAL DE ACUERDO CON LOS TUTORES ACADÉMICOS}

Reconociendo como funciones específicas del tutor académico el seguimiento efectivo de las prácticas laborales, la conexión entre empleador y la Universidad y el control académico y evaluativo de la práctica, el nivel competencial percibido por más del 50\% (Tabla 2.) en Gestión de proyectos, consideran que los retos de aprendizaje que los estudiantes poseen para alcanzar ese dominio, van ajustados al ciclo formativo y la realidad empresarial de los estudiantes y que la falta de aplicabilidad de información constituyen motivos suficientes 
para esta deficiencia de dominio. La formación con la que vienen de la institución poco tiene que ver con la realidad. Sólo se les enseña lo que van a necesitar en su trabajo diario. Un 5\% conceptúan que no hay un dominio competencial de resolución de problemas, mientras un $77 \%$ creen que solo bajamente alcanzan a identificar problemas y a resolverlos. Se argumenta que la aplicación efectiva para resolver problemas no se adaptaba a la realidad del proceso de práctica, y no se valora el esfuerzo, tiempo y dedicación que se debe poner en la actividad de aprendizaje del estudiante y a su reconocimiento y valoración explícitamente por parte del empleador.

Tabla 2. Distribución porcentual de las respuestas dadas en el cuestionario por los empresarios, tutores de práctica y estudiantes

\begin{tabular}{|c|c|c|c|c|c|}
\hline Competencia & Rango & $\begin{array}{l}\text { Nivel de } \\
\text { dominio }\end{array}$ & $\begin{array}{l}\text { Porcentaje } \\
\text { percepción } \\
\text { empresario }\end{array}$ & $\begin{array}{c}\text { Porcentaje } \\
\text { percepción tutor }\end{array}$ & $\begin{array}{c}\text { Porcentaje } \\
\text { autopercepción } \\
\text { estudiante }\end{array}$ \\
\hline $\begin{array}{l}\text { Gestión de } \\
\text { proyectos }\end{array}$ & $\begin{array}{l}0-1 \\
1-2 \\
2-3 \\
3-4\end{array}$ & $\begin{array}{l}\text { Sin dominio } \\
\text { Bajo } \\
\text { Medio } \\
\text { Alto } \\
\end{array}$ & $\begin{array}{l}\text { N/A } \\
86 \% \\
12 \% \\
\text { N/A }\end{array}$ & $\begin{array}{l}14 \% \\
54 \% \\
32 \% \\
\text { N/A }\end{array}$ & $\begin{array}{l}\text { N/A } \\
78 \% \\
22 \% \\
\text { N/A }\end{array}$ \\
\hline $\begin{array}{l}\text { Resolución de } \\
\text { problemas }\end{array}$ & $\begin{array}{l}0-1 \\
1-2 \\
2-3 \\
3-4\end{array}$ & $\begin{array}{l}\text { Sin dominio } \\
\text { Bajo } \\
\text { Medio } \\
\text { Alto }\end{array}$ & $\begin{array}{l}\text { N/A } \\
77 \% \\
23 \% \\
\text { N/A }\end{array}$ & $\begin{array}{c}5 \% \\
77 \% \\
18 \% \\
\text { N/A }\end{array}$ & $\begin{array}{c}\text { N/A } \\
94 \% \\
6 \% \\
\text { N/A }\end{array}$ \\
\hline $\begin{array}{l}\text { Pensar en forma } \\
\text { crítica, lógica y } \\
\text { creativa }\end{array}$ & $\begin{array}{l}0-1 \\
1-2 \\
2-3 \\
3-4\end{array}$ & $\begin{array}{l}\text { Sin dominio } \\
\text { Bajo } \\
\text { Medio } \\
\text { Alto }\end{array}$ & $\begin{array}{l}\text { N/A } \\
12 \% \\
88 \% \\
\text { N/A }\end{array}$ & $\begin{array}{l}\text { N/A } \\
39 \% \\
61 \% \\
\text { N/A }\end{array}$ & $\begin{array}{l}\text { N/A } \\
9 \% \\
91 \% \\
\text { N/A }\end{array}$ \\
\hline $\begin{array}{l}\text { Trabajo } \\
\text { autónomo }\end{array}$ & $\begin{array}{l}0-1 \\
1-2 \\
2-3 \\
3-4\end{array}$ & $\begin{array}{l}\text { Sin dominio } \\
\text { Bajo } \\
\text { Medio } \\
\text { Alto }\end{array}$ & $\begin{array}{c}6 \% \\
87 \% \\
7 \% \\
\text { N/A }\end{array}$ & $\begin{array}{l}9 \% \\
72 \% \\
19 \% \\
\text { N/A }\end{array}$ & $\begin{array}{c}5 \% \\
92 \% \\
3 \% \\
\text { N/A }\end{array}$ \\
\hline $\begin{array}{l}\text { Aprender a } \\
\text { aprender }\end{array}$ & $\begin{array}{l}0-1 \\
1-2 \\
2-3 \\
3-4\end{array}$ & $\begin{array}{l}\text { Sin dominio } \\
\text { Bajo } \\
\text { Medio } \\
\text { Alto }\end{array}$ & $\begin{array}{c}\text { N/A } \\
6 \% \\
94 \% \\
\text { N/A }\end{array}$ & $\begin{array}{l}\text { N/A } \\
81 \% \\
19 \% \\
\text { N/A }\end{array}$ & $\begin{array}{l}\text { N/A } \\
34 \% \\
66 \% \\
\text { N/A }\end{array}$ \\
\hline $\begin{array}{l}\text { Comunicar ideas } \\
\text { de forma efectiva } \\
\text { y otro idioma }\end{array}$ & $\begin{array}{l}0-1 \\
1-2 \\
2-3 \\
3-4\end{array}$ & $\begin{array}{l}\text { Sin dominio } \\
\text { Bajo } \\
\text { Medio } \\
\text { Alto }\end{array}$ & $\begin{array}{l}\text { N/A } \\
86 \% \\
12 \% \\
\text { N/A }\end{array}$ & $\begin{array}{l}9 \% \\
72 \% \\
19 \% \\
\text { N/A }\end{array}$ & $\begin{array}{l}12 \% \\
76 \% \\
12 \% \\
\text { N/A }\end{array}$ \\
\hline Trabajo en grupo & $\begin{array}{l}0-1 \\
1-2 \\
2-3 \\
3-4\end{array}$ & $\begin{array}{l}\text { Sin dominio } \\
\text { Bajo } \\
\text { Medio } \\
\text { Alto }\end{array}$ & $\begin{array}{l}\text { N/A } \\
17 \% \\
83 \% \\
\text { N/A }\end{array}$ & $\begin{array}{l}\text { N/A } \\
12 \% \\
88 \% \\
\text { N/A }\end{array}$ & $\begin{array}{l}\text { N/A } \\
13 \% \\
87 \% \\
\text { N/A }\end{array}$ \\
\hline
\end{tabular}

Fuente: Elaboración propia. 
Si bien, el establecimiento de objetivos concretos como parte de la competencia pensar de forma crítica, lógica y creativa es identificado por un porcentaje alto de tutores (61\%) como dominio medio, la interpretación de los procesos y elaboración coherente de soluciones es aún el "talón de Aquiles" para dominar esta competencia. La poca experiencia laboral de los estudiantes y el poco esfuerzo por aportar ideas frescas, positivas y con valor, implican un desaceleramiento en el dominio competencial visto desde los tutores de práctica.

La responsabilidad adquirida para trabajar de manera autónoma, así como la relación de independencia, el interés propio del practicante por fortalecer, desarrollar o transformar lo aprendido en relación con su práctica ha sido valorado de manera baja por los tutores. El $72 \%$ de ellos cree que el alcance en este proceso no se manifiestan aspectos como la toma de decisiones y la falta de toma de conciencia y control de su propio aprendizaje. En su labor de reconocimiento, el $81 \%$ de los tutores valoran como bajo el dominio competencial de los estudiantes en relación con la competencia de aprender a aprender; consideran que los estudiantes aún no disponen de las de habilidades para iniciarse en la práctica y su capacidad de aprendizaje no traduce eficacia y autonomía, todavía no integran los conocimientos necesarios para para aplicarlos.

Cuando se habla de la competencia comunicar ideas de forma efectiva y otro idioma, el $72 \%$ de los entrevistados apuntan que el dominio de esta competencia solo alcanza un bajo nivel, pues la falta de reconocimiento de las potencialidades propias de comunicación que los estudiantes poseen, carece de efectividad y se destaca la falta de provecho y voluntad para superar estos inconvenientes, y así progresivamente aumentar su propia seguridad para comunicar ideas.

El 88\% de tutores académicos dieron argumentos de aceptación media del dominio competencial del trabajo en grupo, donde la implicación del trabajo colaborativo, la toma de decisiones y las habilidades de capacidad analítica y crítica velaron por relación positiva por parte de este agente participante.

\subsection{NIVEL DE AUTOPERCEPCIÓN COMPETENCIAL DE ACUERDO CON LOS ESTUDIANTES}

Mediante la autoevaluación los estudiantes pueden reflexionar y reconocer sus propios aprendizajes. En este sentido, demuestran como intervienen en su práctica laboral. Al contrastar el nivel de aprendizaje con los dominios competenciales esperados se pudo detectar que, con referencia a la competencia gestión de proyectos, un $78 \%$ reconocen un dominio bajo, en el que consideran que aún encuentran dificultades para la identificación y la entrega de resultados en plazos establecidos, de igual forma la toma de acciones para corregir su desempeño con las responsabilidades en su gestión merecería un poco más de reconocimiento y planificación. No obstante, un $22 \%$ consideran que poseen un dominio medio relacionado con su trabajo sistemático y ordenado para gestionar proyectos particulares.

Un 94\% de los estudiantes se autoevalúan en la competencia resolución de problemas con un bajo dominio y el $6 \%$ restante creen poseer un dominio medio. Frente a esto, la forma de plantear estratégicamente, la toma de decisiones de los estudiantes ante problemas y los planes de acción para contratacarlos, se convierten en la parte más difícil que los implica. También, parece manifestarse al indicar un miedo a afrontar situaciones nuevas, rechazo, fracaso y nuevos retos. 
Un 91\% indicó poseer un dominio medio con la competencia pensamiento crítico, lógico y creativo, esto posiblemente ligado a la avalancha de información que se está generando actualmente y a la que tienen acceso constantemente los estudiantes. Según ellos se debe a la mezcla de pensamiento crítico y creativo que les permite diferenciar lo relevante de lo irrelevante. También se argumentaron que su pensamiento crítico y lógico los hace individuos más hábiles, y por lo tanto están constantemente estructurando y reforzando procesos creativos.

La competencia trabajo autónomo fue valorada con un $92 \%$ de bajo dominio y con un $5 \%$ de absoluta falta de dominio. Los estudiantes no han generado un interés adecuado por el fortalecimiento de sus habilidades autónomas de trabajo, antagónico a la forma cómo piensan y ven el mundo en una era tecnológica. La misma oleada de información ha impedido el desarrollo de esta habilidad, destacan. 66\% reconocen que el dominio de la competencia aprender a aprender es medio. Está ligado al compromiso que ellos han construido a partir de los conocimientos y habilidades que han adquirido en la práctica laboral y a sus experiencias académicas, donde están comprometidos a reutilizarlos en los contextos prácticos para integrar y elaborar modelos de aprendizaje.

La comunicación de ideas de forma efectiva y otro idioma, es considerada por un $76 \%$ como dominio bajo, debido al desconocimiento de respuestas a preguntas específicas de la práctica laboral. Por otro lado, la constante emisión y recepción de información en la práctica laboral y la falta de control de los elementos que intervienen en una comunicación profesional, generan mal entendidos y conflictos, eso también ligado al desconocimiento total o parcial de otra lengua (12\%).

Un $87 \%$ de los practicandos afirman poseer un dominio medio de la competencia trabajo en equipo, destacando el constante acercamiento como una necesidad de tener relaciones con los miembros de su contexto de práctica y de complementariedad para lograr retos que no han podido alcanzar cuando trabajan como autónomos. Cabe notar que aseguran que han creado lazos de cohesión, compañerismo y sentido de pertenencia con la empresa donde realizan la práctica. Por otro lado, han proclaman el interés por el trabajo por logro de objetivos comunes y el establecimiento de relaciones laborales positivas.

\subsection{COMPARACIÓN DEL NIVEL DE RELACIÓN DE COMPETENCIAS POR TODOS LOS AGENTES DE PARTICIPACIÓN}

Los resultados comparados por la media en las competencias de estudio son muy similares entre los tres grupos de informantes (Figura 3.).

En lo que respecta a la relación entre las respuestas de cada colectivo es destacable, como existe homogeneidad entre el grupo dado que el valor de la desviación típica (Figura 3), es siempre inferior a uno.

En la competencia gestión de proyectos, resalta un nivel bajo, consensuado, por los tres informantes, si bien, este nivel se puede comprender por las limitaciones que los estudiantes pueden encontrar cuando se establecen relaciones entre la identificación, el análisis, la toma de decisiones y planes de acción ante un problema que en la práctica es novedoso para ellos, al igual que sucede con la limitación competencial y habilidad para emitir juicios sobre la manera como estas se relacionan (Figura 4.).

Por otra parte, se vuelve a corroborar homogeneidad entre informantes dado que la desviación típica es inferior a uno en todos los casos, además, en la competencia pensar de 
forma lógica, crítica y creativa el valor de la desviación típica es muy pequeño por lo que parece que se produce consenso.

Figura 3. Medias y desviación típica por competencia

\begin{tabular}{|c|c|c|c|c|c|c|c|c|c|c|c|c|c|c|c|c|c|c|c|c|c|c|}
\hline \multirow{3}{*}{$\begin{array}{l}\text { PRIORIDAD } \\
\text { DOMINIO }\end{array}$} & & \multicolumn{21}{|c|}{ COMPETENCIA } \\
\hline & & \multicolumn{3}{|c|}{$\begin{array}{l}\text { Gestión de } \\
\text { proyectos }\end{array}$} & \multicolumn{3}{|c|}{$\begin{array}{l}\text { Resolución de } \\
\text { problemas }\end{array}$} & \multicolumn{3}{|c|}{$\begin{array}{l}\text { Pensar en forma } \\
\text { critica, lógica y } \\
\text { creativa }\end{array}$} & \multicolumn{3}{|c|}{ Trabajo autónomo } & \multicolumn{3}{|c|}{ Aprender a aprender } & \multicolumn{3}{|c|}{$\begin{array}{c}\begin{array}{c}\text { Comunicar ideas de } \\
\text { forma efectiva y otro } \\
\text { idioma }\end{array} \\
\end{array}$} & \multicolumn{3}{|c|}{ Trabajo en grupo } \\
\hline & & EM & $T$ & ES & EM & $T$ & ES & EM & $T$ & ES & EM & $T$ & ES & EM & $T$ & ES & EM & $T$ & ES & EM & $T$ & ES \\
\hline \multirow{2}{*}{\multicolumn{2}{|c|}{$\begin{array}{l}\text { PRIO 1 } \\
\text { BÁSICO }\end{array}$}} & 2.3 & 2.0 & 2.04 & 1.6 & 1.4 & 2.4 & 1.77 & 1.6 & 2.4 & 1.7 & 2.0 & 1.9 & 3.1 & 2.9 & 3.0 & 1.8 & 1.9 & 2.0 & 3.1 & 3.3 & 3.0 \\
\hline & $s_{x}$ & 0.833 & 0.943 & 0.709 & 0.467 & 0.558 & 0.774 & 0.661 & 0.805 & 0.550 & 0.438 & 0.476 & 0.256 & 0.305 & 0.167 & 0.630 & 0.401 & 7.54 & 0.567 & 0.378 & 0.756 & 0.456 \\
\hline \multirow{2}{*}{$\begin{array}{l}\text { PRIO } 1 \\
\text { MEDIO }\end{array}$} & $x$ & 3.1 & \begin{tabular}{|l|l|}
3.4 & \\
\end{tabular} & 3.3 & 1.6 & 2.3 & 1.2 & 3.0 & \begin{tabular}{|l|l|}
3.1 & \\
\end{tabular} & 1.2 & 3.1 & 2.9 & 3.0 & 3.2 & 2.9 & 2.9 & 3.1 & 2.8 & \begin{tabular}{|l|}
2.9 \\
\end{tabular} & 3.2 & 3.3 & 3.0 \\
\hline & $s_{x}$ & 0.798 & 0.609 & 0.762 & 0.467 & 0.793 & 0.439 & 0.426 & 0.662 & 0.701 & 0.504 & 0.795 & 0.232 & 0.450 & 0.167 & 0.319 & 0.401 & 0.303 & 0.280 & 0.439 & 0.549 & 0.576 \\
\hline \multirow{2}{*}{$\begin{array}{l}\text { PRIO 1 } \\
\text { AVANZADO }\end{array}$} & $x$ & 3.4 & 3.3 & 3.5 & 3.2 & 3.1 & 3.2 & 3.1 & 3.2 & 3.2 & 2.9 & \begin{tabular}{|l|l|}
2.6 & \\
\end{tabular} & 2.7 & 3.3 & 2.5 & 2.9 & 3.2 & 2.6 & \begin{tabular}{|l|}
3.0 \\
\end{tabular} & 3.1 & 3.3 & 3.0 \\
\hline & $f^{3}$ & 0.631 & 0.504 & 0.618 & 0.637 & 0.762 & 0.637 & 0.762 & 0.797 & 0.624 & 0.734 & 0.675 & 0.336 & 0.487 & 0.500 & \begin{tabular}{|l|}
0.319 \\
\end{tabular} & 0.434 & 0.566 & \begin{tabular}{|l|}
0.602 \\
\end{tabular} & 0.487 & 0.624 & 0.570 \\
\hline \multirow{2}{*}{$\begin{array}{l}\text { PRIO 2 } \\
\text { BÁSICO }\end{array}$} & $x$ & 2.0 & \begin{tabular}{|l|}
1.8 \\
\end{tabular} & 2.6 & 1.2 & 1.7 & 1.3 & 2.1 & 1.8 & 1.3 & 1.8 & 2.1 & 2.5 & 2.0 & 2.4 & 2.4 & 1.9 & 2.0 & 2.4 & 1.8 & 2.1 & 2.0 \\
\hline & $s_{x}$ & 0.549 & 0.805 & 0.607 & 0.422 & 0.832 & 0.494 & 0.538 & 0.648 & 0.507 & 0.526 & 0.621 & 0.280 & 0.572 & 0.646 & \begin{tabular}{|l|}
0.378 \\
\end{tabular} & 0.612 & 0.624 & \begin{tabular}{|l|}
0.319 \\
\end{tabular} & 0.463 & 0.539 & 0.378 \\
\hline \multirow{2}{*}{$\begin{array}{l}\text { PRIO } 2 \\
\text { MEDIO }\end{array}$} & $x$ & 3.2 & 2.8 & 2.1 & 1.2 & 2.1 & 1.5 & 3.2 & 3.0 & 1.5 & 3.0 & 2.6 & 2.1 & 2.0 & 2.5 & 3.0 & 3.3 & \begin{tabular}{|l|}
2.7 \\
\end{tabular} & 2.0 & 3.1 & 3.0 & 2.9 \\
\hline & $s_{x}$ & 0.646 & 0.932 & 0.604 & 0.422 & 0.683 & 0.504 & 0.454 & 0.502 & 0.703 & 0.630 & 0.531 & 0.390 & 0.567 & 0.632 & \begin{tabular}{|l|} 
\\
\end{tabular} & 0.526 & \begin{tabular}{|l|}
0.453 \\
\end{tabular} & \begin{tabular}{|l|}
0.830 \\
\end{tabular} & 0.493 & 0.283 & 0.280 \\
\hline \multirow{2}{*}{$\begin{array}{l}\text { PRIO } 2 \\
\text { AVANZADO }\end{array}$} & $\bar{x}$ & 3.4 & 3.2 & 3.5 & 3.1 & 2.8 & 2.9 & 3.1 & 3.1 & 2.9 & 3.3 & 2.7 & 2.1 & 3.1 & 2.3 & 3.0 & 3.2 & 2.7 & \begin{tabular}{|l|}
1.9 \\
\end{tabular} & 3.2 & 2.6 & 2.7 \\
\hline & $s_{x}$ & 0.576 & 0.670 & 0.529 & 0.609 & 0.683 & 0.500 & 0544 & 0.710 & 0.236 & 0.616 & 0.618 & 0.243 & 0.347 & 0.721 & \begin{tabular}{|l|}
0.444 \\
\end{tabular} & 0.459 & 0.481 & 0.500 & 0.589 & 0.493 & 0.454 \\
\hline \multirow{2}{*}{$\begin{array}{l}\text { PRIO } 3 \\
\text { BÁSICO }\end{array}$} & $\bar{x}$ & 1.6 & 2.1 & 1.7 & \begin{tabular}{|l|l}
1.7 \\
\end{tabular} & 1.6 & 1.7 & 2.3 & 2.7 & 1.7 & 2.5 & 2.7 & 1.9 & 2.7 & 2.3 & \begin{tabular}{|l|}
3.0 \\
\end{tabular} & 1.5 & \begin{tabular}{|l|}
1.7 \\
\end{tabular} & \begin{tabular}{|l|}
1.4 \\
\end{tabular} & 1.4 & 1.3 & \begin{tabular}{|l|}
1.7 \\
\end{tabular} \\
\hline & $s_{x}$ & 0.487 & 1.167 & 0.454 & 0.453 & 0.738 & 0.508 & 0.446 & 0.403 & 0.360 & 0.697 & 0.701 & 0.506 & 0.615 & 0.426 & 0.611 & 0.504 & 0.434 & 0.378 & 0.497 & 0.477 & 0.337 \\
\hline \multirow{2}{*}{$\begin{array}{l}\text { PRIO } 3 \\
\text { MEDIO }\end{array}$} & $x$ & 2.6 & 3.2 & 2.8 & 3.2 & 2.8 & 3.1 & 3.0 & 2.6 & 3.1 & 3.1 & 2.9 & 3.0 & 3.2 & 2.7 & 3.1 & 2.7 & \begin{tabular}{|l|}
2.7 \\
\end{tabular} & \begin{tabular}{|l|}
2.6 \\
\end{tabular} & 3.2 & 3.4 & \begin{tabular}{|l|}
2.2 \\
\end{tabular} \\
\hline & $s_{x}$ & 0.645 & 0.797 & 0.593 & 0.439 & 0.830 & 0.486 & 0.426 & 0.487 & 0.458 & 0.465 & 0.674 & 0.500 & 0.428 & 0.715 & 0.454 & 0.496 & 0.358 & 0.295 & 0.711 & 0.649 & 0.225 \\
\hline \multirow{2}{*}{\begin{tabular}{l|} 
PRIO 3 \\
AVANZADO
\end{tabular}} & $\bar{x}$ & 3.1 & 2.8 & 3.0 & 3.3 & 3.1 & 2.6 & 3.2 & 2.8 & 2.6 & 3.0 & 2.7 & 2.4 & 3.2 & 2.4 & 2.2 & 3.2 & 2.8 & 2.8 & 3.6 & 3.3 & 3.5 \\
\hline & $s_{x}$ & 0.609 & 0.507 & 0.554 & 0.590 & 0.820 & 0.399 & 0.640 & 0.622 & 0.288 & 0.649 & 0.513 & 0.558 & 0.599 & 0.806 & 0.409 & 0.478 & \begin{tabular}{|l|}
0.542 \\
\end{tabular} & 0.167 & 0.407 & 0.543 & 0.232 \\
\hline \multirow{2}{*}{\multicolumn{2}{|c|}{$\begin{array}{l}\text { PRIO } 4 \\
\text { BÁSICO }\end{array}$}} & 1.6 & 1.7 & 1.5 & \begin{tabular}{|l|l|}
1.6 \\
\end{tabular} & 1.9 & 1.9 & 1.8 & \begin{tabular}{|l|}
1.9 \\
\end{tabular} & 1.9 & 2.6 & 2.7 & 2.9 & 2.6 & 1.4 & \begin{tabular}{|l|}
1.9 \\
\end{tabular} & 3.0 & 2.9 & \begin{tabular}{|l|}
2.1 \\
\end{tabular} & N/A & N/A & N/A \\
\hline & & 0.487 & 0.806 & 0.504 & 0.478 & 0.842 & 0.272 & 04.55 & 0.604 & 0.197 & 0.688 & 0.637 & 0.280 & 0.537 & 0.548 & 0.279 & 0.305 & 0.605 & 0.280 & N/A & N/A & $N / A$ \\
\hline \multirow{2}{*}{\multicolumn{2}{|c|}{$\begin{array}{l}\text { PRIO } 4 \\
\text { MEDIO }\end{array}$}} & 1.6 & 2.3 & 1.8 & 3.1 & 2.7 & 2.5 & 3.1 & \begin{tabular}{|l|}
3.0 \\
\end{tabular} & 2.5 & 3.0 & 3.0 & 3.0 & 3.1 & \begin{tabular}{|l|}
2.3 \\
\end{tabular} & \begin{tabular}{|l|}
1.9 \\
\end{tabular} & 3.1 & \begin{tabular}{|l|}
2.9 \\
\end{tabular} & \begin{tabular}{|l|}
2.4 \\
\end{tabular} & N/A & N/A & N/A \\
\hline & $s_{x}$ & 0.487 & 0.494 & 0.319 & 0.351 & 0.458 & 0.626 & 0.319 & 0.697 & 0.351 & 0.630 & 0.475 & 0.280 & 0.305 & 0.513 & 0.401 & 0.292 & \begin{tabular}{|l|}
0.630 \\
\end{tabular} & 0.232 & & & $\mathrm{~N} / \mathrm{A}$ \\
\hline \multirow{2}{*}{$\begin{array}{l}\text { PRIO } 4 \\
\text { AVANZADO }\end{array}$} & $\bar{x}$ & 3.7 & 3.3 & 3.6 & 3.4 & 2.9 & 3.0 & 3.2 & 3.2 & 3.0 & 3.3 & 3.1 & 2.3 & 3.1 & 2.3 & 2.4 & 3.1 & 2.4 & 2.5 & N/A & N/A & N/A \\
\hline & $s_{x}$ & 0.454 & 0.749 & 0.577 & 0.506 & 0.673 & 0.543 & 0.439 & 0.861 & 0.280 & 0577 & \begin{tabular}{|l|l|}
0.543 \\
\end{tabular} & 0.599 & 0.401 & 0.542 & 0.409 & 0.351 & 0.557 & 0.428 & N/A & $N / A$ & N/A \\
\hline
\end{tabular}

Nota: PRIO: Prioridad, EM: Empresario, T: Tutor, ES: Estudiante, N/A: No aplica.

Fuente: Elaboración propia.

Así, en la competencia resolución de problemas, (Figura 4.), nuevamente el nivel es muy bajo y puede deberse a la inexperiencia del practicante al identificar posibles problemas, sus causas y las soluciones. Hay homogeneidad entre los participantes debido a que la desviación típica es menor a uno en todas las situaciones. 
Figura 4. Medias de prioridades competencias

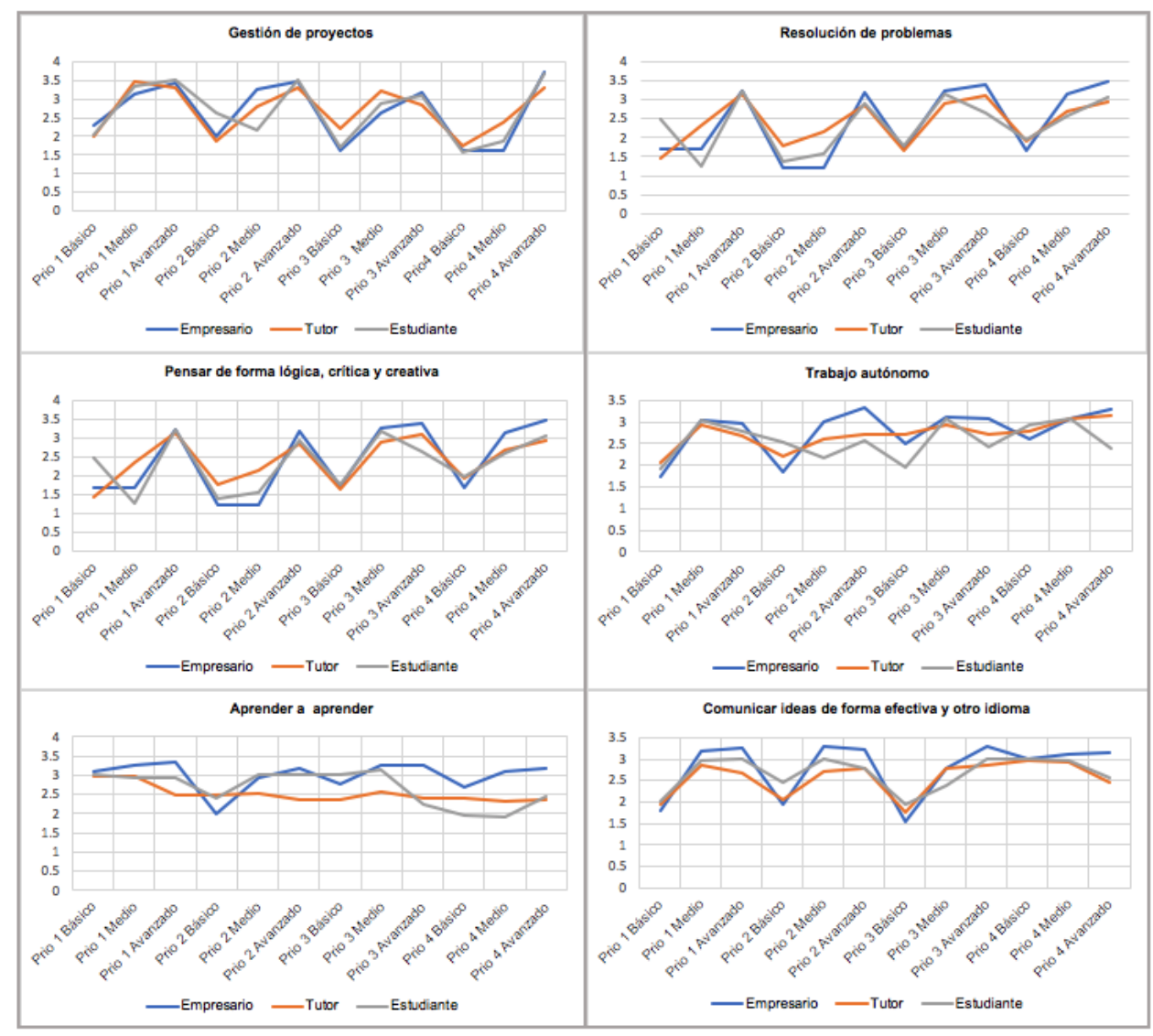

Fuente: Elaboración propia.

En cuanto a las competencias pensar de forma lógica, crítica y creativa, trabajo autónomo, aprender a aprender y trabajo en equipo (Figura 5.), permiten comprobar como los diferentes colectivos establecen valores de dominio muy similares y continúa habiendo homogeneidad.

Es importante identificar que las medias que los estudiantes expresan en la competencia comunicar ideas de forma efectiva y otro idioma, son más bajas que el resto de los grupos. Posiblemente influenciadas por el desconocimiento de otro idioma, los niveles de interacción en la comunicación en el lugar de trabajo y por la misma inexperiencia que frena o acelera los niveles de comunicación.

Por último, comparando la puntación media dada en todas las competencias por los grupos, la valoración de los estudiantes es casi siempre superior que la de los empresarios y tutores, especialmente en los niveles avanzados como en gestión de proyectos con una media de 3.6. No obstante, la diferencia no es pequeña siendo siempre inferior a 3.3 y 3.2 respectivamente. 
Figura 5. Medias de prioridades competencia trabajo en equipo



Fuente: Elaboración propia.

\section{DISCUSIÓN Y CONCLUSIONES}

El estudio desarrollado ha permitido establecer una falta de desinformación por parte de los estudiantes frente al dominio de sus propias competencias. Las competencias seleccionadas para este estudio se caracterizan por ser esencialmente evolutivas y cambiantes a lo largo de la práctica. La implicación de evaluación empresarial, académica y una autoevaluación permite obtener una apreciación de los logros personales en términos de los criterios individuales y así identificar los aspectos a mejorar (Ramírez García et al., 2014). Es importante considerar el beneficio de implementar mecanismos que los guíe a mejorar y dominar los niveles de sus competencias en la práctica laboral de manera gradual hasta que ellos sepan valorar su propio quehacer y el grado de satisfacción competencial. Ante el bajo dominio competencial en la praxis demostrado por los resultados ofrecidos por empresarios y tutores académicos, e incluso por los autoevaluados por los estudiantes, es necesario que la coordinación de prácticas de la Universidad ejecute una estrategia de acompañamiento para los estudiantes desde el inicio de su práctica hasta el final. Estas tácticas de acompañamiento deben estar encaminadas hacia el entrenamiento para mejorar cada una de las competencias seleccionadas. Hacia el incremento del dominio de la competencia gestión de proyectos, una planificación estratégica como alternativa, podría incluir proyectos de mejora de la calidad de procesos académicos, y la formación de practicantes autónomos y capaces de dirigir y procesar sus acciones formativas de calidad en aprendizaje (Tinoco-Giraldo, 2018). Así como también, impulsar la gestión por competencias como un proceso que permita la identificación las capacidades y habilidades necesarias para los puestos de trabajo ofrecidos en la práctica empresarial a través de la creación de perfil profesional en el área de mercadeo que sea cuantificable y medible objetivamente. Diseñar e implementar un plan de mejora que permita potenciar las competencias profesionales de los estudiantes próximos a realizar 
la práctica con la intención de ofrecerles herramientas que les permitan el reconocimiento ágil del pensamiento crítico, lógico y creativo. Importante también es destacar que para la realización de tareas como trabajado autónomo se requieren ciertas habilidades y capacidades, que propicien el mejoramiento del proceso de enseñanza-aprendizaje por lo que la formación convierte en una condición sine qua non de esta sugerencia (Espinoza-Freire et al., 2017). Velar por enfatizar esfuerzos en la competencia de comunicación de ideas de forma efectiva y otro idioma. Se deben potenciar esfuerzos y aumentar lazos de atracción interpersonal, fijar una serie de normas que dirijan a los estudiantes a invertir suficiente tiempo para aprender o al menos a adquirir cierto dominio de otro idioma de forma profesional.

Es fundamental promover la práctica de la buena comunicación entre los empleadores, tutores y estudiantes y trabajar por el logro de los objetivos comunes y establecer relaciones positivas. Por último, resulta indispensable implementar un nuevo estilo de participación empresa-academia que permita gestionar los procesos de práctica de una manera más integral y efectiva de acuerdo con las líneas estratégicas actuales.

\section{REFERENCIAS BIBLIOGRÁFICAS}

Alcaraz Espín, J. J., Bel Sala, M., Briones Peñalver, A. J., Egea López, E., García Cascales, M. S., García Martín, A., Molina García-Pardo, J. M., Gómez López, D., Illán Gómez, F., Lozano Gutiérrez, M... \& Segado, I. (2013). Desarrollo de competencias profesionales a través de prácticas en empresa en la universidad politécnica de Cartagena (UPCT). Equipos Docentes: Innovación Docente en la Universidad Politécnica de Cartagena, 63-103. Recuperado de http:// repositorio.upct.es/xmlui/bitstream/handle/10317/3658/edi.pdf?sequence=1\&isAllowed=y

Álvarez Pérez, P. R., \& González Alfonso, M.C. (2005). La tutoría entre iguales y la orientación universitaria: una experiencia de formación académica y profesional. Revista Educar, (36), 107128. Recuperado de https://www.raco.cat/index.php/Educar/article/view/48984/49873

Álvarez-Santullano, M. \& De Prada Creo, E. (2018). Evaluación de las competencias profesionales a través de las prácticas externas: Incidencia de la creatividad. Revista de Investigación Educativa, 36(1), 203-219. doi: https://doi.org/10.6018/rie.36.1.275651

Arias, I. M. (2015). Diseño y validación de un cuestionario de escala formativa para valorar las competencias transversales de los estudiantes universitarios. Una propuesta para dispositivos móviles basada en Android. Universitas Tarraconensis. Revista de Ciències de l'Educació, 1(1), 84-87. doi: https://doi.org/10.17345/ute.2015.1.661

Aznar-Díaz, I., De la Cruz, R. R. \& Romero-Rodríguez, J. M. (2018). Implicación de las empresas en la formación profesional para el empleo. Análisis sobre la idoneidad de la selección de sus acciones formativas. European Scientific Journal, ESJ, 14(10), 18-31. doi: http://dx.doi. org/10.19044/esj.2018.v14n10p18

Bermúdez, A., García Varea, I., López, M. T., Montero, F., Ossa, L. D. L., Puerta, J. M., Rojo, T. \& Sánchez, J. L. (2011). Una Definición precisa del concepto nivel de dominio de una competencia en el marco del aprendizaje basado en competencias. JENUI 2011: XVIII Jornadas de Enseñanza Universitaria de la Informática, 169-176. Recuperado de https://idus.us.es/xmlui/bitstream/ handle/11441/61501/a20.pdf?sequence=1\&isAllowed=y

Bogdan, J. (2018). Club Management: Practicum: A Case Study in Evidence that Practicums are "Better" than Internships. 2018 International Summit, 22-38. doi: https://doi.org/10.25101/18.18

Bozal, M. G. (2006). Escala mixta Likert-Thurstone. ANDULI, Revista Andaluza de Ciencias Sociales, (5), 81-95. Recuperado de https://revistascientificas.us.es/index.php/anduli/article/ view/3728/3256 
Callahan, G. \& Benzing, C. (2004). Assessing the role of internships in the career-oriented employment of graduating college students. Education \& Training, 46(2), 82-89. doi: https:// doi.org/10.1108/00400910410525261

Camacho, I. (2018). Mentoría en educación superior, la experiencia en un programa extracurricular. Revista Electrónica de Investigación Educativa, 20(4), 86-99. doi: https://doi.org/10.24320/ redie.2018.20.4.1999

Cano García, M. E. (2008). La evaluación por competencias en la educación superior. Profesorado. Revista de Curriculum y Formación de Profesorado, 12(3), 1-16. Recuperado de https://www. redalyc.org/pdf/567/56712875011.pdf

Carrera Erazo, S., Parreño Sánchez, J. \& Ayala Esparza, V. (2018). El desempeño profesional de los estudiantes universitarios en las prácticas pre-profesionales. Opuntia Brava, 9(1), 89-96. Recuperado de http://opuntiabrava.ult.edu.cu/index.php/opuntiabrava/article/view/120

Casal, J. \& Mateu, E. (2003). Tipos de muestreo. Rev. Epidem. Med. Prev, 1(1), 3-7.

Ceja Oseguera, S., De la Torre Hidalgo, T. L. \& Ramírez Murillo, L. M. (2018). Análisis de las competencias genéricas que se desarrollan con el uso de las redes sociales. Un estudio en el área de negocios de la Universidad Popular Autónoma del Estado de Puebla. Red Internacional de Investigadores en Competitividad, 5(1). Recuperado de https://riico.net/index.php/riico/article/ view/635/1229

Charria Ortiz, V. H., Sarsosa Prowesk, K. V., Uribe Rodríguez, A. F., López Lesmes, C. N. \& Arenas Ortiz, F. (2011). Definición y clasificación teórica de las competencias académicas, profesionales y laborales. Las competencias del psicólogo en Colombia. Psicología desde el Caribe, (28), 133-165. Recuperado de https://www.redalyc.org/html/213/21320758007/

Chávez, F. H., Cantú, M. \& Rodríguez, C. M. (2016). Competencias digitales y tratamiento de información desde la mirada infantil. Revista Electrónica de Investigación Educativa, 18(1), 209-220. Recuperado de http://redie.uabc.mx/redie/article/view/631

Dreyfus, H. L., \& Dreyfus, S. E. (2005). Peripheral vision: Expertise in real world contexts. Organization Studies, 26(5), 779-792. doi: https://doi.org/10.1177/0170840605053102

Espinoza-Freire, E., Serrano Polo, O., \& Brito Paredes, P. (2017). El trabajo autónomo en estudiantes de la Universidad Técnica de Machala. Revista Universidad y Sociedad, 9(2), 202-212. Recuperado de https://rus.ucf.edu.cu/index.php/rus/article/view/569

Estrada, M. \& John, H. (2012). La formación por competencias y el mundo del trabajo: de la calificación a la empleabilidad. Revista de Salud Pública, 14, 98-111. Recuperado de https:// www.redalyc.org/pdf/422/42223293009.pdf

Fernández, J. T. \& Bueno, C. R. (2015). Evaluación de competencias profesionales en educación superior: retos e implicaciones. Revista Educación XX1, 19(1). doi: https://doi.org/10.5944/ educxx 1.12175

Lentzen, S. (2016). Los modelos de formación dual como integración de lo académico y lo laboral. Revista Prácticum, 1(1). Recuperado de https://revistapracticum.com/index.php/iop/ article/view/2/41

Ley General de Educación. Ministerio de Educación Nacional de Colombia. [MEN]. (febrero 8, 1994). Bogotá 1994, Recuperado el 10 de agosto de 2014, de http://www.alcaldiabogota.gov.co/ sisjur/normas/Norma1.jsp?i=292

Machado Ramírez, E. F. \& Montes de Oca Recio, N. (2009). El desarrollo de habilidades investigativas en la educación superior: la solución de problemas profesionales. Humanidades Médicas, 9(2), 1-21. Recuperado de http://scielo.sld.cu/scielo.php?pid=S1727$81202009000200002 \&$ script=sci_arttext\&tlng=pt

Marín, R., Guzmán, I. \& Castro, G. (2012). Diseño y validación de un instrumento para la evaluación de competencias en preescolar. Revista Electrónica de Investigación Educativa, 14(1), 182-202. Recuperado de http://redie.uabc.mx/vol14no1/contenido-maringuzmanc.html

Martínez Carrillo, C. A. \& Peña Pinzón, F. A. (2013). Pertinencia e impacto de las prácticas 
empresariales desde la perspectiva de los empresarios, graduados y estudiantes en práctica de la Universidad Pontificia Bolivariana Seccional Bucaramanga. Recuperado de https://repository. upb.edu.co/bitstream/handle/20.500.11912/1278/digital_19952.pdf?sequence=1\&isAllowed=y

Medina Rivilla, A. M. \& Domínguez Garrido, M. C. (2006). Los procesos de observación del prácticum: análisis de las competencias. Revista Española de Pedagogía, 64(233), 69-103. Recuperado de https://dialnet.unirioja.es/servlet/articulo?codigo=1973266

Montoya, C. (2009). Evaluación del desempeño como herramienta para el análisis del capital humano. Revista Científica "Visión de Futuro", 11(1). Recuperado de https://www.redalyc.org/ pdf/3579/357935472005.pdf

Navarro Soria, I., González Gómez, C., López Monsalve, B. \& Botella Pérez, P. (2015). Aprendizaje de contenidos académicos y desarrollo de competencias profesionales mediante prácticas didácticas centradas en el trabajo cooperativo y relaciones multidisciplinares. Revista de Investigación Educativa, 33(1), 99-117. doi: http://dx.doi.org/10.6018/rie.33.1.183971

Proyecto Tuning. (2006). Una introducción a: Tuning Educational Structures in Europe. La contribución de las universidades al proceso de Bolonia. Recuperado de http://www.unideusto. org/tuningeu/images/stories/Publications/ENGLISH_BROCHURE_FOR_WEBSITE.pdf

Ramírez García, A., Corpas Reina, C., Amor Almedina, M. I. \& Serrano Rodríguez, R. (2014). ¿De qué soy capaz? Autoevaluación de las competencias básicas. Revista Electrónica de Investigación Educativa, 16(3), 33-53. Recuperado de http://www.scielo.org.mx/scielo.php?pid=S160740412014000300003\&script=sci_arttext

Romero-Martín, M. R., Castejón-Oliva, F. J., López-Pastor, V. M. \& Fraile-Aranda, A. (2017). Evaluación formativa, competencias comunicativas y TIC en la formación del profesorado. Comunicar, 52(XXV), 73-82. Recuperado de http://rabida.uhu.es/dspace/bitstream/ handle/10272/14089/Evaluacion-formativa.pdf?sequence $=2$

Saldarriaga Ríos, J. G., Martínez Gómez, J. \& Restrepo Múnera, M. L. (2016). La formación en los postgrados en administración: Desarrollo de competencias para la investigación. Revista Espacios, 37(10), E-1. Recuperado de http://www.revistaespacios.com/a16v37n10/163710e1.html

Schambach, T. P. \& Dirks, J. (2002). Student perceptions of internship experiences. Paper presented at the 17th Annual Conference of the International Academy for Information Management, Barcelona, Spain, 1-8. Retrieved from https://files.eric.ed.gov/fulltext/ED481733.pdf

Tejada Fernández, J. (2005). El trabajo por competencias en el prácticum: cómo organizarlo y cómo evaluarlo. Revista electrónica de Investigación educativa, 7(2), 1-31. Recuperado de http:// www.scielo.org.mx/pdf/redie/v7n2/v7n2a13.pdf

Tinoco-Giraldo, H. (2018). Diseño de un programa de e-mentoring a partir del análisis de competencias adquiridas por los estudiantes de práctica empresarial del programa de mercadeo. Lúmina, (19), 48-70. doi: http:dx.doi.org/10.30554/lúmina.19.2782.2018

Valencia, J. C. (2018). La didáctica de la investigación, una mirada a una escuela de administración de empresas. Revista Lúmina, (19), 128-151. doi: http:dx.doi.org/10.30554/lúmina.19.2600.2018

Véliz Martínez, P. L., Jorna Calixto, A. R. \& Berra Socarrás, E. M. (2016). Consideraciones sobre los enfoques, definiciones y tendencias de las competencias profesionales. Educación Médica Superior, 30(2). Recuperado de http://scielo.sld.cu/scielo.php?script=sci_arttext\&pid $=$ S0864-21412016000200018

Zabalza, M. A. (2016). El prácticum y las prácticas externas en la formación universitaria. Revista Prácticum, 1(1), 1-23. Recuperado de https://revistapracticum.com/index.php/iop/article/ view/15/42 